\title{
Malaria vaccines
}

At a conservative estimate there are about 100 million cases of malaria in the world each year. ${ }^{1}$ Recent community based studies in West Africa give credence to the oft quoted, but previously poorly substantiated, estimate of a million childhood deaths a year in sub-saharan Africa alone. ${ }^{2}$ Malaria, particularly Plasmodium falciparum, remains a major killer of the world's children. The postwar successes in eradicating malaria from a number of temperate and subtropical areas reached a limit set not only by logistic problems, escalating costs, and growing insecticide resistance but also by natural epidemiological factors. Malaria could never have been eradicated from its heartland in tropical Africa, even with the optimum use of the measures available at that time. The present situation is not even stable. The combination of rapidly emerging multidrug resistance, social and political dislocation in some countries, and unplanned urbanisation in others is a cause for real worry. New approaches are needed to bolster currently available control measures and an antimalarial vaccine has become the holy grail of malaria research.

\section{What kind of malaria vaccine?}

Currently most effort is being directed at developing vaccines against three separate stages of the parasite's life cycle.

(1) The sporozoite-an effective vaccine would prevent entry of the infective stage into the liver and thus block subsequent development.

(2) The blood stage-in this case sporozoites would enter the liver and develop normally but an effective vaccine would prevent the subsequent rapid expansion of blood stages and thus limit clinical illness.

(3) The sexual stage-an effective vaccine would not protect the recipient against illness but would render the sexual forms of the parasite, which appear during the later stages of a blood infection, unable to infect mosquitoes. Such an 'altruistic' vaccine would be transmission blocking and would be used as an adjunct to other control measures in specific epidemiological settings.

The ideal vaccine would, of course, be cheap, completely safe, and totally effective! What constitutes a useful vaccine, however, will depend on the specific requirements of the group being vaccinated.
This annotation is mostly concerned with children in Africa, who stand in greatest need and also present the most difficult challenge. For this group a vaccine will certainly need to be cheap and safe but it will also have to give longlasting protection against clinical disease and be available in formulations capable of being used in existing vaccination schedules. In other areas of the tropics, particularly parts of South East Asia, the balance between malaria and current control measures is such that a vaccine conferring little clinical protection but giving good transmission blocking immunity may be enough to tip the balance against the parasite. Finally there is a large group of non-immune expatriates who visit endemic areas for short or medium terms. For this group risk benefit analysis is important, as a vaccine which gives even short term protection to only some recipients may have uses if it is safe. The attractiveness of such an adjunct to available measures might increase as the effectiveness of available prophylactic drugs decreases.

\section{Rationale for a human malarial vaccine}

What are the grounds for believing that vaccination is an achievable aim? Firstly, people do become immune to clinical malaria-the emergence of stable societies in Africa would not have been possible if that were not the case. In highly endemic areas children suffer the brunt of morbidity and mortality; adults become functionally immune to symptomatic disease but not to low level parasitisation. Secondly, it is clear from many animal models that vaccination with whole parasites can produce varying degrees of immunity. An important point to emerge from such studies is that immunity is both species and stage specific - that is, successful vaccination with sporozoites will protect against sporozoite challenge but not against the direct innoculation of blood stage parasites and vice versa. These background facts have been clear for many years but the problems of ever obtaining antigenic material from the different parasite stages in either sufficient quantity or of sufficient purity seemed unsurmountable. The arrival on the scene of monoclonal and recombinant DNA technology changed all that.

\section{Progress so far}

Most work is orientated towards vaccines against $P$ 
falciparum because it is the major killer, but work on vaccines against $P$ vivax is also gaining momentum. For all three parasite stages the general strategy is the same; antigenic analysis of the parasite and identification of antigens capable of inducing potentially protective immunological responses, cloning and sequencing the genes for such 'protective' antigens, and then production either by recombinant DNA technology or artificial synthesis of subunit vaccines.

To date most progress has been made with the antisporozoite vaccine. Sporozoites have a surface coat consisting of a single polypeptide, the circumsporozoite protein. Genes for the circumsporozoite protein of a number of malarial species have been cloned. Antibodies capable of blocking the penetration of sporozoites into hepatocytes turn out to be directed towards a region of multiple tandem repeats of short runs of amino acids. The repeat motifs are characteristic for each malarial parasite. Most importantly, in the case of $P$ falciparum the simple repeat structure, based on just four amino acids, turns out to be invariant between all strains so far examined. Two experimental vaccines based on the repeat sequence, one synthesised and one produced by recombinant technology, have recently undergone preliminary trials in human volunteers. ${ }^{34}$ In both trials there was a degree of protection against sporozoite challenge in some vaccine recipients. Responses to vaccination, however, showed wide variation and were poorly sustained and evidence for protection was based mainly on relatively small increases in time to patent parasitaemia from challenge. In each trial one individual was apparently completely protected but the numbers involved are too small to bear further analysis. Some commentators have been disappointed by these results, others have been elated by the success. In reality both responses are appropriate, the failures do show how much remains to be done and some of the problems are discussed below. But this should not detract from the real achievement, the idea that subunit vaccines can under certain circumstances protect against human malaria has been strengthened and this should give encouragement to all working on vaccines against other stages and other protozoan parasites.

The position with regard to blood stage vaccines is more complicated. Many blood stage antigens have now been characterised and in several cases the genes have been cloned. Some antigens turn out to exhibit marked antigenic diversity, making them unsuitable as vaccine candidates. In other cases defining epitopes capable of inducing protection has not been straightforward. However, two blood stage antigens (known by their relative molecular mass) are at an advanced stage of investigation. Pf195 is a quantitatively major glycoprotein on the surface of intracellular erythrocytic schizonts. During red cell rupture and reinvasion the protein undergoes processing to several smaller molecules forming part of the merozoite's surface coat. Immunisation of mice with the analogous protein of a rodent malaria resulted in impressive protection ${ }^{5}$ though vaccination of monkeys with affinity purified $\mathrm{Pf} 195^{6}$ or synthetic peptides representing parts of the processed molecule ${ }^{7}$ have produced only modest partial protection. The second antigen, Pf155 (also known as RESA-ring infected erythrocyte surface antigen) is released from merozoites during red cell invasion and is incorporated into host red cell membranes. The gene for Pf155 has been cloned and sequenced and initial vaccine trials in monkeys with recombinant proteins corresponding to various regions of the molecule have given encouraging results. ${ }^{8}$ It seems likely that human trials of blood stage vaccines based on one or both of these molecules will begin within the next year or two. Antigametocyte vaccines have tended to be less 'news worthy' probably because they do not offer the possibility of individual protection. Nevertheless recent progress has been rapid and several candidate antigens have been identified.

\section{The remaining problems}

The main technical problems still to be overcome, well illustrated by the antisporozoite vaccine, are how to induce strong, longlasting responses in all vaccinees. There are two vital areas, firstly the development of acceptable and effective adjuvants and secondly the definition of epitopes capable of inducing $\mathrm{T}$ helper responses. The next generation of experimental antisporozoite vaccines will probably incorporate sequences representing such epitopes from within the circumsporozoite protein. There is a lurking fear here, that it would be ironic if natural $\mathrm{T}$ cell epitopes turned out to lie in regions of the molecule capable of exhibiting the greatest diversity. Putting these worries aside, the optimistic view is that it will prove possible to arrive at effective synthetic vaccines, probably incorporating several different antigens. We will then be faced with the problems of testing candidate vaccines. Why should this be problematic? Two aspects can be illustrated; firstly malarial epidemiology has been traditionally based on the use of cross sectional malariometric indices such as parasite rates and spleen rates. These have been used to generate models centred on the parasitisation of communities. The primary aim of vaccination, however, will not be to eradicate parasitisation per se but to limit morbidity and 
mortality. The factors governing the relation between parasitisation and disease are not understood. What is clear is that traditional malariometric tools will not be adequate for monitoring vaccine trials. The only acceptable end point of a vaccine trial is a reduction in deaths and severe disease and this will necessitate very large trials. This is not a fundamental problem, epidemiologists are used to the idea of big trials but it does mean that a whole new set of epidemiological tools will have to be developed for detecting and measuring malaria specific morbidity and mortality under difficult field conditions. A second problem arises from the fact that an essential part of any large scale trial will be monitoring the effect of an intervention on the one thing which does already protect people from malaria, the development of natural immunity. Unfortunately work on naturally acquired immunity to malaria has lagged behind the more basic aspects of malarial research with the result that we simply do not yet know how to measure immunity to malaria. It is clear that new initiatives to stimulate malarial field research will be required before the anticipated fruits of the last decade's advances can be realised. Finally, it has to be noted that many children continue to die from diseases for which good preventative measures already exist. The successful field testing of a malarial vaccine would in many ways mark the beginning of the most difficult part of the story.

\section{Conclusions}

Developments to date give rise to cautious optimism. Considerable problems remain but they can probably be overcome. So how far away are we from having a useful vaccine? The answer to this question depends to some extent on what you want one for. It is not inconceivable that vaccines suitable for use as adjuncts to other prophylactic measures in subjects with limited exposure will be available over a five to ten year time span. But for children in the tropics and particularly in sub-saharan Africa it is not likely that malarial vaccines will have any perceptable impact before the end of the century. By that time another 10 to 20 million children will have died of malaria. A malaria vaccine is not just around the corner for them, but it has probably moved to the right side of the horizon.

\section{References}

1 Tropical disease research: a global partnerhsip. Eighth programme report. UNDP/World Bank/WHO special programme for research and training in tropical diseases. Geneva: World Health Organisation, 1987.

2 Greenwood BM, Bradley AK, Greenwood AM, et al. Mortality and morbidity from malaria among children in a rural area of The Gambia, West Africa. Trans $R$ Soc Trop Med Hyg 1987;81:478-86.

${ }^{3}$ Ballou WR, Hoffman SL, Sherwood JA, et al. Safety and efficacy of a recombinant DNA Plasmodium falciparum sporozoite vaccine. Lancet 1987;i:1277-81.

4 Herrington DA, Clyde DF, Losonsky G, et al. Safety and immunogenicity in man of a synthetic peptide malaria vaccine against P falciparum sporozoites. Nature 1987;328:257-9.

5 Holder AA, Freeman RR. Immunization against blood-stage rodent malaria using purified parasite antigens. Nature 1981 ;294:361-4

${ }^{6}$ Hall R, Hyde JE, Goman M, et al. Major surface antigen gene of a human malaria parasite cloned and expressed in bacteria Nature 1984;311:379-82.

7 Patarroyo M, Romero P. Torres ML, et al. Induction of protective immunity against experimental infection with malaria using synthetic peptides. Nature 1987;328:629-32.

${ }^{8}$ Collins WE, Anders RF, Pappaioanu M, et al. Immunization of Aotus monkeys with recombinant proteins of an erythrocytc surface antigen of Plasmodium falciparum. Nature 1986;323: 259-62.

K MARSH
University of Oxford,
Nuffield Department of Clinical Medicine,
John Radcliffe Hospital,
Headington
Oxford OX3 9DU

